\title{
Psoriasis Skin Models as Promising Tools in Psoriasis Research
}

\author{
InJae Jeong, Hee Jung Lee* \\ Department of Dermatology, CHA University, Korea
}

Received: February 01, 2018; Published: February 13, 2018

*Corresponding author: Hee Jung Lee, Department of Dermatology, CHA University, Korea, Tel: 82-31-780-5240; Fax: 82-31-780-5247; Email: derma97@gmail.com

\begin{abstract}
Psoriasis is a common immune-mediated chronic inflammatory skin disease. Several cytokines involved in the immunological mechanism of psoriasis have now been identified. Known cytokines are entangled to form a large signalling pathway and are currently being studied to find cytokines that play the most important role in pathogenesis. Psoriasis signalling mechanisms have been known through various studies using cells, mouse models, and psoriasis skin models. The psoriasis skin model is an important tool for psoriasis studies. This review summarizes the psoriasis skin model currently in use.
\end{abstract}

Keywords: Psoriasis; Skin equivalent; Skin model

\section{Introduction}

Psoriasis is a common immune-mediated chronic inflammatory disease that affects approximately $2 \sim 3 \%$ of the worldwide population. One of the pathogeneses of psoriasis is genetic components, and many genes are associated with it, but it is unclear how those genes work together [1]. Over recent years, there have been great advances on $\mathrm{T}$ cells and their roles in the inflammatory and autoimmune diseases that help us understand more deeply the pathogenesis of psoriasis. These immune cells move from the dermis to the epidermis and secrete inflammatory cytokines such as tumor necrosis factor- $\alpha$ (TNF- $\alpha$ ), interleukin-1 (IL-1), interleukin-6 (IL-6), interleukin-17 (IL-17) and interleukin-22 (IL-22). These secreted inflammatory signals stimulate the keratinocytes to proliferate and differentiate. Understanding these signaling pathways have led to the development of biologic agents that target cytokines and are now being used in clinical practice [2,3]. Some of the psoriasis signaling processes has been known through studies using the psoriasis skin model. Currently, researchers are continuously studying the pathogenesis of disease and therapeutic targets by reproducing the disease by in vitro and in vivo psoriasis skin model $[4,5]$. Here, we review the various current psoriasis skin models and summarize the advantages, disadvantages and suitability for describing certain aspects of psoriasis.

\section{In vitro Models}

In vitro model is relatively easy to obtain, cost-effective, and free from ethical and immunological problems developed in animal models. Recent advances in tissue engineering have enabled development of in vitro reconstructed human epidermis models to mimic normal stratified human epidermal morphology [6]. The limitations of the in vitro psoriasis skin model are the absence of blood vessels and microenvironment. Nevertheless, in vitro psoriasis skin model is useful for investigating pathogenic mechanisms of the disease.

\section{Two-dimensional (2D) psoriasis skin model}

Two-dimensional epidermal culture systems have been widely used to reveal pathologic mechanism and therapeutic target of psoriasis because they can be easily reproduced. An initial attempt to generate in vitro model of psoriasis was performed using skin cells from newly developed psoriasis skin lesion. The cultivation of keratinocytes derived from psoriasis patient showed drawback such as lack of microenvironment, reduced expression of psoriasis-related genes, and growth retardation. Thus, normal human epidermal keratinocytes (NHEK) and immortalized human keratinocytes line (HaCaT) are frequently used. In this case, cytokines that are well-known to induce psoriasis are added to the cell lines in order to reproduce psoriasis-like keratinocytes layers [7]. Currently, the optimal mixture of cytokines to produce the psoriasis skin model includes IL-1, IL-6, IL-17A, IL-22, TNF- $\alpha$ and IFN- $\gamma$. Recent data showed that cytokine-treated keratinocytes exhibited a similar system in which the inflammatory markers are up-regulated $[8,9]$. Co-culture systems have long been used 
to study cell-cell interactions. A co-culture model of keratinocytes and fibroblasts can be established to mimic the in vivo psoriatic microenvironment more closely. The co-culture skin model of psoriasis has shown that one cytokine secretion can affect the expression of other keratinocytes, leading to a feedback loop that appears in psoriasis skin. The use of co-culture has been able to explore the specific role of other cell types in this way and reveal the mechanism of cell-cell interactions [10].

\section{Three-dimensional (3D) psoriasis skin model}

Two-dimensional cell culture cannot completely replace normal skin structure composed of epidermis and dermis and is not suitable for experiments using microenvironments. Threedimensional cell culture through the use of human biopsied tissue will be more appropriate because it indicates the signaling pathway and pathogenic mechanisms present in the psoriasis. However, this model is limited by the availability, diversity, and ethical issues of skin donors. To solve this problem, more advanced systems such as three-dimensional tissue engineering human skin equivalents have been introduced into the studies.3D skin equivalent scan be simply divided as epidermal equivalents containing multilayered epidermis, and full thickness skin equivalents containing epidermal and dermal skin components [11]. When reconstructing skin artificially, it is important to develop methods for mass production with low costs that are capable of keratinization through terminal differentiation. Several methods have been studied that induce differentiation of keratinocytes to reproduce the stratum corneum, including adjusting calcium and serum concentrations of culture media, air-exposure, and mechanical stress.

Normal 3D skin equivalent models are commercially available these days, but psoriatic epidermal models are limited. However, with the addition of a cytokine cocktail, normal 3Dskin equivalent can adapt a psoriasis-like phenotype and gene expression profile. Previous studies revealed that IL-22 or a mixture of IL17, IL-22 and TNF- $\alpha$ triggered hyperplasia in normal 3D skin equivalent, with a reduced expression of differentiation-related genes and up regulation of psoriasis markers [12]. Full thickness skin equivalents, which have functional dermal matrix and fibroblasts, are more similar to human skin. In order to generate full thickness skin equivalents, several matrices can be used such as porous membranes, de-epidermises dermis and fibroblastcontaining dermal scaffolds. To generate psoriatic full thickness skin equivalents, the influence of keratinocytes or fibroblasts from psoriasis involved skin was assessed previously. Although diseased cells have the intrinsic capacity to form the psoriasis phenotype, their use is limited by the scarcity and heterogeneity of patientderived cells. Therefore, studies are focusing on the direction of healthy cells to a diseased state using inflammatory cytokines, similar to the epidermal skin equivalents.

\section{In Vivo Models}

In vivo studies are often used to explain the pathogenesis of disease and to test the efficacy of a new therapeutic agent. Unlike mouse skin model, the human dermis is thick and the epidermis consists of stratified layers, and there is a difference in the immunological mechanism involved. So far, in vivo mouse model cannot be considered a model that accurately reflects the disease [13]. Nevertheless, the in vivo mouse model has been used to greatly expand the current knowledge of psoriasis causes, etiologies, and treatments. In vivo mouse models of psoriasis are divided into spontaneous, genetically engineered, xeno transplantation and direct induction $[14,15]$.

\section{Spontaneous mutation model}

There are almost 100 mouse mutations that result in phenotypic characteristics of psoriasis, such as hyperkeratosis or psoriatic formation. However, as T cells do not influx the skin in this model, they are often used as models to study specific aspects of psoriasis such as hyperkeratosis, neutrophil influencing, and angiogenesis. Homozygous ( $\left.\mathrm{Scd} 1^{\mathrm{ab}} / \mathrm{Scd} 1^{\mathrm{ab}}\right)$ mice were the first in vivo models of hyperkeratosis. Reduction of sebaceous glands is a characteristic of mutations in the Scd1 gene. The loss of the sebaceous glands mice were the first in vivo model of psoriasis [16]. Second, the mutation used in the spontaneous in vivo model is flaky skin mice $\left(\mathrm{Ttc}^{\mathrm{fs}} /\right.$ $\mathrm{Ttc}^{\mathrm{fsn}}$ ). It induces the hyperkeratosis and inflammation of epithelia from the moment of birth and represents a Koebner reaction.

\section{Genetic engineered model}

Advances in molecular biology have enabled the generation of mouse models based on the over expression or loss of function of selected genes and their products. Transgenic animals have been developed that target specific factors or signaling pathways. Using a genetically modified mouse model, it was possible to clarify the relationship and role of specific cytokines, growth factors, and mediators of the inflammatory response in psoriasis outbreaks. The genes involved in the differentiation and proliferation of lymphocytes and keratinocytes were targeted and knock-out mice were produced. Initially, leukocyte 2 integrands or proteins involved in the differentiation of lymphocytes and neutrophil such as c-Jun and Jung were targeted. Currently, knock-out mice targeting cytokines known as key players in the signal transduction of psoriasis, such as IL-1, IL-17A, TNF- $\alpha$ and IFN- $\gamma$, are made. In addition, vascular endothelial growth factor associated with angiogenesis and keratinocytes growth factor associated with keratinocytes differentiation have been used as target genes $[17,18]$.

\section{Xeno-transplantation model}

Xeno-transplantation is another approach to developing in vivo models of the disease. Xeno-transplantation is a method of transplanting psoriasis patients' skin or equivalents made from in vitro culture models into immune deficient mice. Mice with severe combined immunodeficiency syndromes (SCIDs) are widely used as models in psoriasis studies. After grafting the psoriasis patient's skin to the nude mouse, the grafts maintained the psoriatic histology features for more than 2 months [19]. However, the major limitation of in vivo SCIDs mouse model is the presence of neutrophil and mature natural killer cells. AGR129 mice have mutations in IFN receptor type 1, 2 and recombines enzyme activation gene-2.As a 
result of this mutation, mature natural killer cell as well as $\mathrm{T}$ cell remains inactive, which facilitates the graft of psoriasis patient skin. Interestingly, transplanting the skin of non-lesion sites of psoriasis patients into AGR129 mice results in spontaneous plaque formation without the injection of cytokines associated with psoriasis. Despite a number of limitations, xeno-transplantation is one of the best in vivo psoriasis skin models because it mimics almost all the spectrum of psoriasis phenotype [20].

\section{Direct Induced Model}

Patients using imiquimod reported the appearance of psoriasis lesions. Imiquimod increases the activation of Th1 responses and migration of Langerhans cells through the activation of ligands to Toll-like receptors in macrophages, mononuclear cells and plasmacytoid dendrite cells. Using this mechanism, imiquimod was continuously applied to the mouse skin to develop a psoriasis skin model that could reproduce the immunological mechanism that occurred in psoriasis lesions [21]. Recently, in vivo psoriasis skin model was developed by intradermal injecting cytokines related to the signal transduction of psoriasis such as IL-17A, IL-23 and TNF- $\alpha$ into mouse skin. This affects the differentiation and proliferation of keratinocytes, resulting in psoriatic histological changes such as hyperkeratosis, parakeratosis and acanthosis [22].

\section{Conclusion}

Psoriasis is a chronic skin disease with a variety of clinical features ranging from mild to severe. A variety of studies have revealed the signaling pathway associated with psoriasis, including studies with psoriasis skin models, and key players involved in signal transduction such as IL-17, IL-23 and TNF- $\alpha$ are known. Target therapeutic agents that inhibit the key players have been developed and are currently in clinical practice. However, there is no drug that can completely resolve psoriasis, and the treatment response varies depending on the patients. A psoriasis skin model may help to solve this problem. The psoriasis skin model not only helps to discover new substances in preclinical laboratory phages, but also helps to measure side effects and effects in clinical trial phases. However, currently available in vivo and in vitro psoriasis skin models can't fully reproduce psoriasis. Evolution of the psoriasis skin model is essential to increase the success rate of new target therapy development and reduce time and cost. Herein, in addition to studies on the signaling pathway through existing psoriasis skin models, studies on psoriasis skin models that can better implement psoriasis should be conducted concurrently.

\section{References}

1. Nestle FO, Kaplan DH, Barker J (2009) Psoriasis. N Engl J Med 361(5): 496-509.

2. Kagami S, Rizzo HL, Lee JJ, Koguchi Y, Blauvelt A (2010) Circulating Th17, Th22, and Th1 cells are increased in psoriasis. J Invest Dermatol 130(5): 1373-1383.

3. Luan L, Ding Y, Han S, Zhang Z, Liu X (2014) An increased proportion of circulating Th22 and Tc22 cells in psoriasis. Cell Immunol 290(2): 196200.
4. Naves LB, Dhand C, Almeida L, Rajamani L, Ramakrishna S (2016) In vitro skin models and tissue engineering protocols for skin graft applications. Essays Biochem 60(4): 357-369.

5. Wagner EF, Schonthaler HB, Guinea Viniegra J, Tschachler E (2010) Psoriasis what we have learned from mouse models. Nat Rev Rheumatol 6(12): 704-714.

6. Bracke S, Desmet E, Guerrero Aspizua S, Tjabringa SG, Schalkwijk J, et al. (2013) Identifying targets for topical RNAi therapeutics in psoriasis: assessment of a new in vitro psoriasis model. Arch Dermatol Res 305(6): 501-512.

7. Dallaglio K, Marconi A, Truzzi F, Lotti R, Palazzo E, et al. (2013) E-FABP induces differentiation in normal human keratinocytes and modulates the differentiation process in psoriatic keratinocytes in vitro. Exp Dermatol 22(4): 255-261.

8. Chiricozzi A, Guttman Yassky E, Suarez Farinas M, Nograles KE, Tian S, et al. (2011) Integrative responses to IL-17 and TNF-alpha in human keratinocytes account for key inflammatory pathogenic circuits in psoriasis. J Invest Dermatol 131(3): 677-687.

9. Nograles KE, Zaba LC, Guttman Yassky E, Fuentes Duculan J, Suarez Farinas M, et al. (2008) Th17 cytokines interleukin (IL)-17 and IL-22 modulate distinct inflammatory and keratinocyte-response pathways. Br J Dermatol 159(5): 1092-1102.

10. Martin G, Guerard S, Fortin MM, Rusu D, Soucy J, et al. (2012) Pathological crosstalk in vitro between $\mathrm{T}$ lymphocytes and lesional keratinocytes in psoriasis: necessity of direct cell to cell contact. Lab Invest 92(7): 10581070.

11. Sidgwick GP, McGeorge D, Bayat A (2016) Functional testing of topical skin formulations using an optimised ex vivo skin organ culture model. Arch Dermatol Res 308(5): 297-308.

12. Chiricozzi A, Nograles KE, Johnson Huang LM, Fuentes Duculan J, Cardinale I, et al. (2014) IL-17 induces an expanded range of downstream genes in reconstituted human epidermis model. PLoS One 9(2): e90284.

13. Tjabringa G, Bergers M, Van Rens D, de Boer R, Lamme E, et al. (2008) Development and validation of human psoriatic skin equivalents. Am J Pathol 173(3): 815-823.

14. Danilenko DM (2008) Review paper preclinical models of psoriasis. Vet Pathol 45(4): 563-575.

15. Nakajima K, Sano S (2017) Mouse models of psoriasis and their relevance. J Dermatol

16. Boehncke WH (2005) The psoriasis SCID mouse model a tool for drug discovery? Ernst Schering Res Found Workshop 50: 213-234.

17. Johnston A, Fritz Y, Dawes SM, Diaconu D, Al Attar PM, et al. (2013) Keratinocyte over expression of IL-17C promotes psoriasiform skin inflammation. J Immunol 190(5): 2252-2262.

18. Bochenska K, Smolinska E, Moskot M, Jakobkiewicz Banecka J, Gabig Ciminska M (2017) Models in the Research Process of Psoriasis. Int J Mol Sci 18(12): 2514.

19. Zollner TM, Renz H, Igney FH, Asadullah K (2004) Animal models of T-cell-mediated skin diseases. Bioessays 26(6): 693-696.

20. Lowes MA, Suarez Farinas M, Krueger JG (2014) Immunology of psoriasis. Annu Rev Immunol 32: 227-255.

21. Van der Fits L, Mourits S, Voerman JS, Kant M, Boon L, et al. (2009) Imiquimod-induced psoriasis like skin inflammation in mice is mediated via the IL-23/IL-17 axis. J Immunol 182(9): 5836-5845.

22. Girolomoni G, Strohal R, Puig L, Bachelez H, Barker J, et al. (2017) The role of IL-23 and the IL-23/TH 17 immune axis in the pathogenesis and treatment of psoriasis. J Eur Acad Dermatol Venereol. 31(10): 16161626. 
(c) (i) This work is licensed under Creative Submission Link: http://biomedres.us/submit-manuscript.php

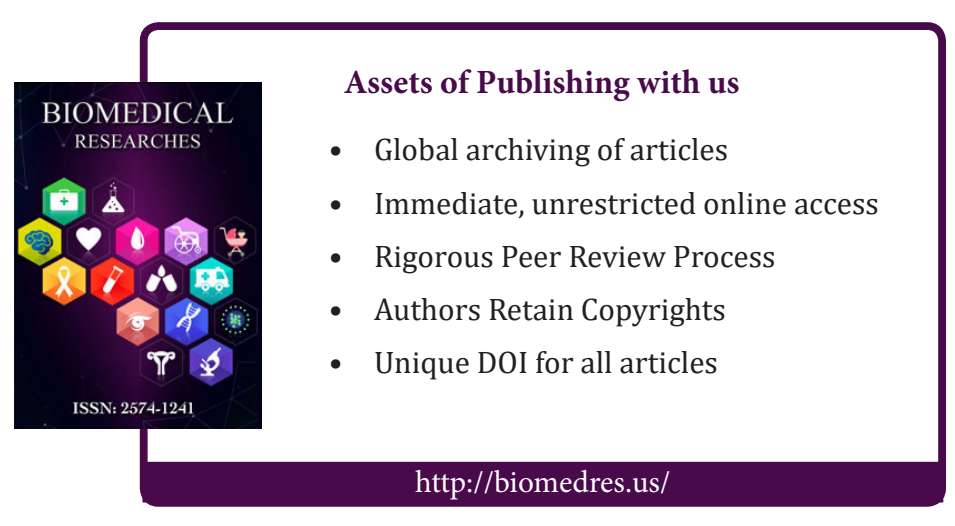

